\title{
Research for Billet Limited Weight Cutting Based on Behavior Stateflow
}

\author{
Wang Fubin, Xu Ao, Zeng Kai, Chen Zhikun, Zhou Yaluo \\ Electrical Engineering College, North China University of Science and Technology. 063009 Tangshan, China
}

\begin{abstract}
It is critical to improve the yield of rolled products and reduce the energy consumption based on billet limited weight cutting. The effect on billet limited weight by dimensional accuracy of billet image and billet casting speed is analyzed, and the behaviour control architecture of billet limited weight cutting is established. The goals of billet limited weight cutting is decomposed into cutting tasks and cutting actions based on finite-state machine theory. The theoretical weight, the integral weight and the predicted weight as the state switching conditions of behaviour control model, so that the billet limited weight cutting is realized. The model of construction is beneficial for the autonomous behaviour control of billet limited weight cutting.
\end{abstract}

\section{Introduction}

It is critical to improve the utilization ratio of steel billet based on billet limited weight cutting. The decrease of billet limited weight cutting error not only can improve the yield of downstream steel rolling process, but also can reduce the energy consumption and the cost of per ton steel, and enhance the economic benefits of enterprise. There are many factors that affect the billet limited weight cutting in the process of steel billet casting, such as the length measurement precision of billet, the speed of billet casting, the wear degree of slab-casting mold, the temperature of casting molten steel, the response sensitivity of cutter clamping and so on. It has high engineering value to improve the accuracy of billet cutting to find the main factors of effecting the billet weight cutting and consider the interactional result of many factors comprehensively, also to promote the intelligent of billet limited weight cutting [1].

This paper analyzed the effective factors of billet limited weight cutting and established the architecture of billet limited weight cutting control system. A finite-state machine behavior logic control strategy of fitting for billet limited weight cutting, which to cut the target decompose step by step for cutting behavior and actions, is proposed. And it explored the autonomous behavior of billet limited weight cutting target.

\section{Parameter Analysis of Stateflow Model for Billet Limited Weight Cutting}

Finite-state machine (FSM) is a discrete system mathematical model, through the realization of state transition in event-driven way. It consists of many state of specific objectives will be completed, trigger events between states and transferred condition. Using the FSM can realize complex logic control function conveniently. Analysis the several main factors of influencing the billet limited weight cutting as follows:

\subsection{Parameter Analysis of Billet Casting Speed}

Many factors that effects the billet limited weight cutting in the process of steel billet casting, such as the speed of billet casting, the temperature of casting molten steel, the wear degree of slab-casting mold and so on. The function relation between the weight of billet and the speed of billet casting can be described as:

$$
G=\sum_{i=1}^{n} S \rho v_{i} \frac{1000}{60}
$$

where, $S$ is the area of billet, $\rho$ is the density of billet, $v_{i}$ is the real-time speed of billet casting.

Through the analysis of a large number of experimental data, it shows that, due to changes in the cross section of billet, the weight of billet and the length of billet appear a phenomenon of dispersion corresponding distribution. Fig.1 illustrates the dispersion relation between weight and length of billet. From Fig.1, the same weight of billet can be corresponding to different range of billet length, the biggest difference between $15 \mathrm{~mm}$. And corresponding to the billet that cross section is $165 \times 165 \mathrm{~mm}$, the error of weight will reach $3.185 \mathrm{~kg}$. If only consider the length of billet as basis in the process of billet cutting, the billet weight will produce a large error. So in the process of billet visual measurement, it can use method of piecewise integral to calculate the weight of billet. This method not only to consider the length of billet, and also consider the cross 
section [2].

Acquisition the speed of billet casting every second, Fig.2 illustrates the statistical distribution between speed of billet casting and the length of billet. From Fig.2, in a certain speed range, the weight range of billet is concentrated. So the speed sum of billet casting is an important factor that affects the weight of billet.

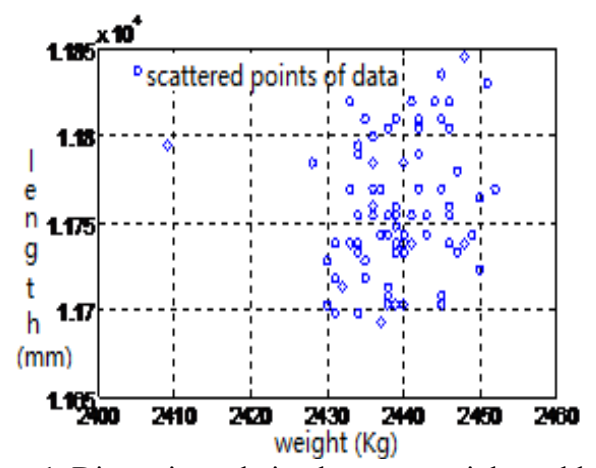

Figure 1. Dispersion relation between weight and length of billet

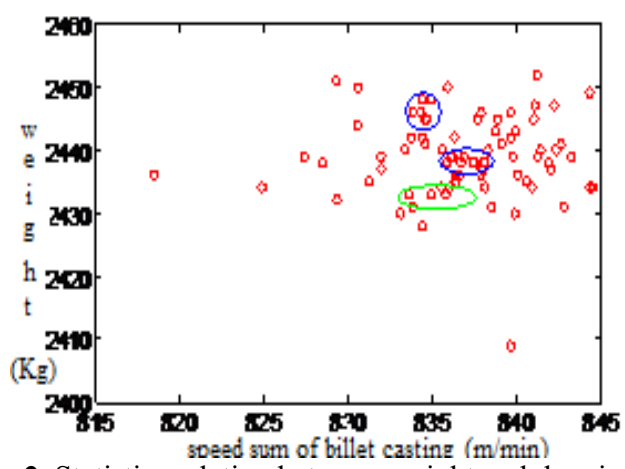

Figure 2. Statistics relation between weight and drawing speed of billet

\subsection{Fourier Transform of Billet Image}

Using the visual measurement to obtain the length of billet, if under the ideal conditions, such as consistent area, stable temperature, and uniform of composition and so on, the length of billet and the weight of billet exists a good linear relationship. However, many factors, such as the speed of billet casting, the length measurement precision of billet and so on, can lead to changes in cross section of billet in the actual process of billet casting. Then these factors can results in a nonlinear corresponding relation between the length of billet and weight of billet. The length measurement precision of billet has a great influence on billet limited weight cutting. When use the visual to measure the length of billet, due to exist the factors such as dust and high temperature turbulence, the images of billet happen degradation, then the factors can effects the segmentation precision and measurement error of images [3].

Using the method of Fourier Transform to filter the original image in the frequency domain. This treatment can remove high the frequency interference and realize image recovery. Then using Fourier inverse transformation to obtain the processing result of image $[4,5]$. Image $f(x, y)$ corresponding to the Fourier spectrum is $F(u, v)$, the two-dimensional discrete Fourier transform of this image can be expressed as:

$$
F(m, n)=\frac{1}{N} \sum_{i=0}^{N-1 N-1} \sum_{k=0}^{N} f(i, k) e^{-j 2 \pi\left(m \frac{i}{N} * n \frac{k}{N}\right)}
$$

The inverse transformation can be expressed as:

$$
f(i, k)=\frac{1}{N} \sum_{i=0}^{N-1 N-1} \sum_{k=0}^{N-1} F(m, n) e^{j 2 \pi\left(m \frac{i}{N} * n \frac{k}{N}\right)}
$$

Fig.3 shows the Fourier transform processing of image, Fig.3(a) is the original image of billet, Fig.3(b) is the discrete Fourier spectrum, Fig.3(c) is the image after discrete Fourier inverse transformation.

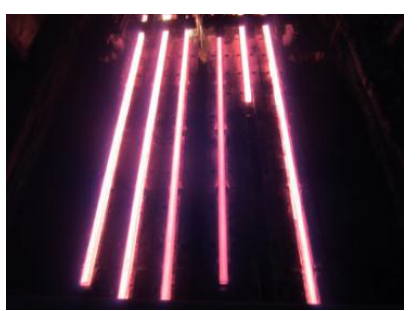

(a)

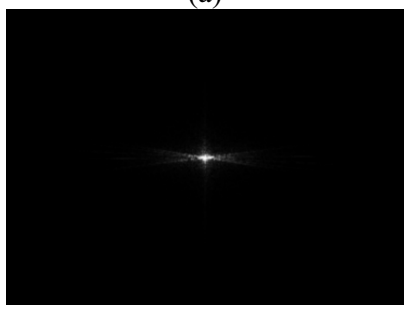

(b)

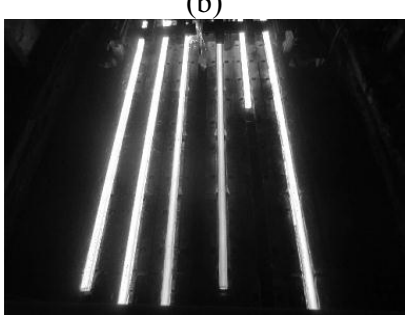

(c)

Figure 3. Discrete Fourier transform for billet image

After Fourier transform, the image can effectively remove noise and improve the reliability of image segmentation, then the visual measurement precision can be improved. The cross section of billet can change because of the wear of slab-casting mold, for small billet. The higher measurement accuracy of visual, the less billet cutting error. So in the process of vision measurement, not only to measure the length of small billet, and also measure the cross sectional area of small billet. Thus the changes of billet weight can be real-time tracking.

\section{Behaviour Stateflow Model of Billet Limited Weight Cutting}

The structure of small billet limited weight cutting is defined a structure model for achieving specific cutting goals by perceiving and processing the information of environment and the control of cutting machine. Behavior 
control have three basic control architectures: deliberating control structure, reaction control structure and mixed control structure. In deliberating control structure, every module is performed sequentially, and each operation should be repeated through planning, decision-making, implementation, so the response of environment is poor; in reaction control structure, task and behavior are separated, specific target task is decomposed into the simple behavior and actions that they can parallel response, so it has a good real-time; mixed control structure is a kind of combination with planning of global goal, decision-making and Reactive task execution.

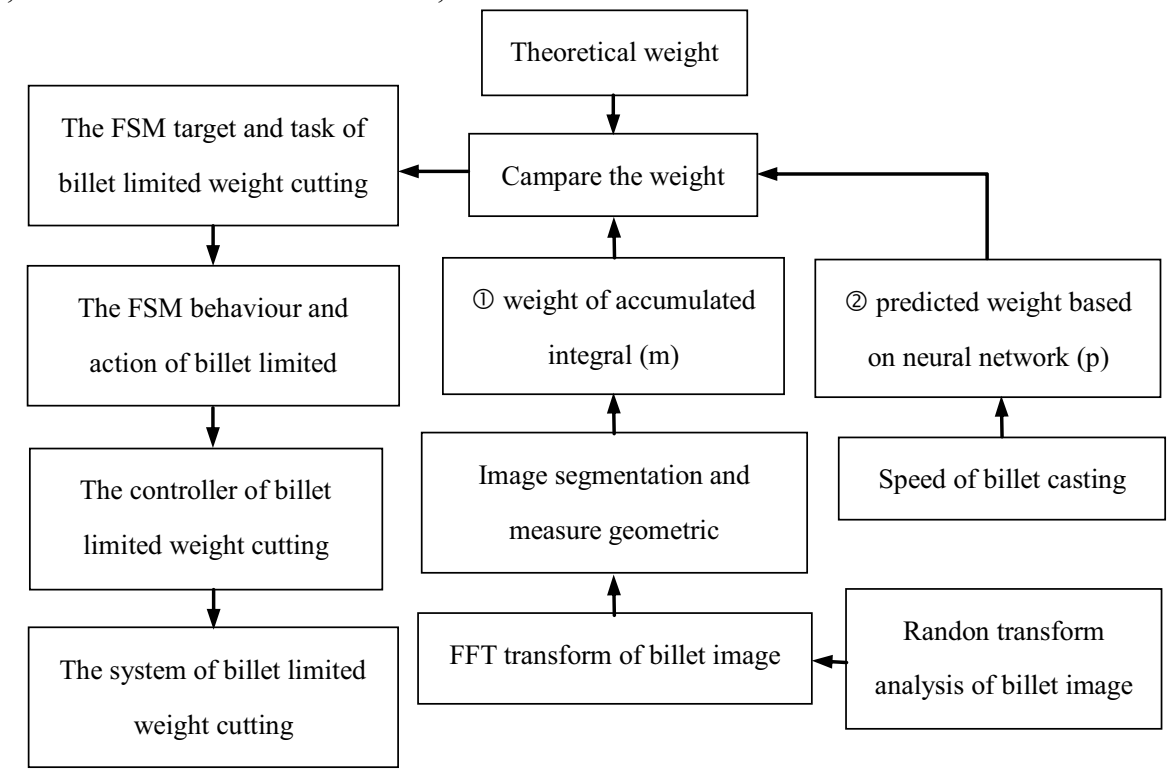

Figure 4. Behavior control architecture for billet weight cutting

Reaction control structure suitable for behavior control of billet limited weight cutting, Fig.4 shows the behavior control architecture of billet limited weight cutting system. It combines perception, planning, decision-making, execution and other links, and it formed a certain intelligence architecture [6,7].

The control system includes modules with measuring perception, planning, decision-making and control. The task plan of limited weight cutting is obtained by FSM, the task of cutting is decomposed into cutter holder control action and flame cutting control action. According to the measured geometric parameter of billet image for real-time accumulated integral the weight of billet, then the weight of billet $P_{1}$ is obtained; at the same time, the speed of billet casting as the input vector, it can forecast the weight of billet, then the weight of billet $P_{2}$ is obtained. The difference between the two weights as the instruction of cutting machine control action, billet limited weight cutting can be realized.

\section{Behavior Stateflow of Weight Cutting Object}

Based on the principle of FSM, make the flame cutting moment of cutting machine as the stable control target, and translate to the control behavior of flame cutting machine. According to the difference value and direction between the forecast weight and the weight of accumulated integral, the action moment of cutting machine is determined, and the control model based on the behavior of FSM is established, also make the different operation state of operation parameter as the event-driven transfer conditions of FSM model.

The state-flow visualization dynamic logical model and simulation toolbox based on FSM are very suitable for establishing and simulating the model of event-driven system and complex logic system [8]. Firstly, creating the model based on the flow diagram, state diagram and graphics functions; then, the theory of finite-state can be realized easily in combination with the event, action of states, action of conditions, transferred action, event broadcast, etc. The generated logic of condition monitoring by state-flow can be connected with the Simulink model by the I/O interface of Chart diagram. So that the behavior control be achieved completely.

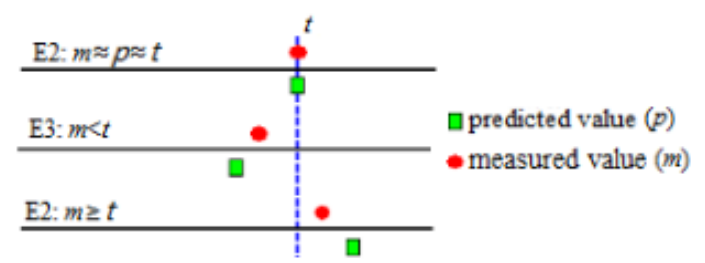

Figure 5. Variable variation of billet weight

Fig.5 illustrates the change process of the theoretical values and the predicted values and the measured values of billet limited weight. Where, $t$ is the theoretical weight value that the ideal length of billet corresponding to billet weight; $m$ is the weight of accumulated integral; $p$ is the predicted weight of billet. For a given billet, the value of $t$ and $p$ are relatively consistent, but $m$ is a real-time variation, so that it can be used as the state transition condition of FSM model. 
Table 1. Finite state and it's transition condition

\begin{tabular}{ccc}
\hline state name & State description & State transfer condition \\
\hline S0 & initial state & E1: start condition \\
S1 & state of normal cutting & E2: the condition of maintaining the normal \\
S2 & predicted value $<$ theoretical value & E3: $p<t, m<t$, when $\mathrm{m} \approx \mathrm{t}$ \\
S3 & predicted value $>$ theoretical value & E4: $p>t$, when $m \geq t$ \\
\hline
\end{tabular}

Table 1 illustrates the various states and the corresponding state transition conditions of FSM model. $\mathrm{E} 1$ is the start condition of system normal operation; E2 is the condition of maintaining the normal cutting that he theoretical value, the forecast value and the measured value are close; E3 is the state of system that he measured values and the predicted value are less than the theoretical value, when the measured value and the theoretical value is closing, trigger the state transition; E4 is state that the predicted value is more than the theoretical value, when the measured value is more than the theoretical value or equal to the theoretical value, trigger the state transition.

When the model of FSM is established, open the figure of Chart into the interface of state diagram editor. According to the theory of FSM, the goal of limited weight cutting can be broken into four states ( $\mathrm{S} 0-\mathrm{S} 3)$, the name of states are listed in table 1. Fig.6 shows the state flow of limited weight cutting.

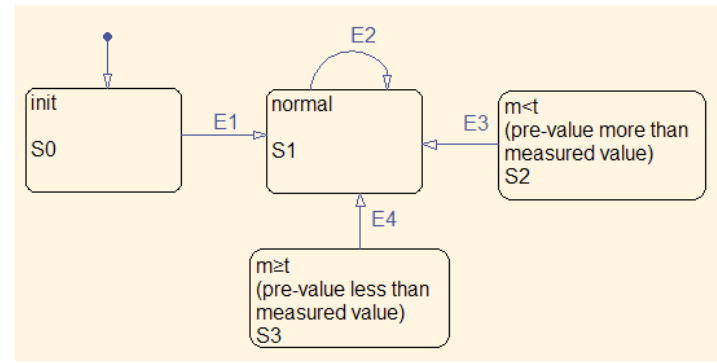

Figure 6. The stateflow for billet weight cutting

The system is in the initial state S0. When the trigger condition E1 of system is meted, it shows that the theoretical value, the forecast value and the measured value are close, so it can perform the task of cutting, in state S1. If the current theoretical value, the measured values and the prediction value is close, also the condition E2 is meted, State S1 is maintained. When the system is in the state S2, it shows that the measured values and the prediction value are less than the theoretical value, the condition E3 is meted, the state happen transition. When the system is in the state S3, it shows that the predicted value is more than the theoretical value, it should wait for the measured values more than or equal to the theoretical value, when the condition E4 is meted, the state happen transition.

\section{Conclusion}

The ability of autonomous behavior control for billet limited weight cutting is the important embodiment of billet cutting system automation level. This paper established behavior control architecture for billet limited weight cutting on the basis of analysis of the steel billet images and analysis of the effect of billet casting speed on billet limited weight cutting. Based on the theory of finite-state machine, achieve behavior state model of billet cutting independently by the stateflow technology. By constructing the transferred condition of different states, achieve transformation between different states in billet cutting. This is a beneficial $\mathrm{e}^{\mathrm{i}}$ exploration for the billet limited weight cutting control method based on behavior.

\section{References}

1. Ge Yadong, Dong Chao. Measures to raise products ratio of bar. Hebei Metallurgy, 12:27-29 (2011)

2. Wang Fubin, Chen Zhikun, Wu Baolin, et al . Forecast for Billet Weight Based on SVM Optimized by Niching Particle Swarm. Control Engineering of China, 22(3):554-558 (2015)

3. Jin Guoping, Xu Fuhao, Wang Yong. Application of the machine vision technology in the billet cut-to-length auto measuring system. Bao-Steel Technology, 3:76-80 (2011)

4. Zhang Chao, Hu Shaohai, Xiao Yang. 2-D DFT-DWT hybrid transform and its application in removing speckle noise for medical ultrasound images . Signal Processing, 25(3): 464-468 (2015)

5. Xiao T., Hu S.H., Xiao Y., 2-D DVF-DWT application to multidimensional signal processing, Proc. ICSP 2006, PP.1306-1309.

6. Jian Liu, Zhong Liu1, Ying Xiong. Method of Parameters Optimization in SVM based on PSO. Transactions on Computer Science and Technology, 2(3):9-16 (2013)

7. Su Mang, Li Fenghua, Shi Guozheng. Action-Based Multi-level Access Control Model. Journal of Computer Research and Development, 51(7):1604-1613 (2014)

8. Mei Yang, Yi Zichen, Wang Lipeng, et al. An improved modulation strategy for the five-leg inverter based on finite state machine 